\title{
Note
}

\section{Etude chromosomique d'un hybride chèvre $\times$ mouton fertile}

\author{
E.P. CRIBIU *, Michèle MATEJKA ${ }^{* *}$, B. DENIS ${ }^{* * *}$ et X. MALHER *** \\ * Institut National de la Recherche Agronomique, Centre de Recherches de Jouy-en-Josas, \\ Laboratoire de Cytogénétique, 78350 Jouy-en-Josas, France \\ ** Ecole Nationale Vétérinaire de Toulouse, Laboratoire de Cytogénétique, \\ Chemin des Capelles, 31076 Toulouse Cedex, France \\ *** Ecole Nationale Vétérinaire de Nantes, Chaire de Zootechnie, BP 527, \\ 44026 Nantes Cedex 03, France
}

\begin{abstract}
Résumé
L'accouplement naturel d'une chèvre avec un bélier a produit une femelle hybride fertile dont le caryotype composé de 57 chromosomes comprend trois autosomes métacentriques. Celle-ci, saillie par un bélier Solognot, a mis bas deux mâles, l'un mort-né et l'autre vivant. Le caryotype de ce dernier était composé de 54 chromosomes.
\end{abstract}

Mots clés : mouton, chèvre, hybride, caryotype.

\section{Summary}

Chromosome study of a fertile female goat $\times$ sheep hybrid

Natural mating of a doe with a ram produced a female hybrid carrying 57 chromosomes, including three metacentric autosomes. This animal backcrossed to a Solognot ram delivered a stillborn and a living male offspring. The karyotype of this last animal consisted of 54 chromosomes.

Key words : goat, sheep, hybrid, karyotype.

\section{Introduction}

Depuis des siècles, les éleveurs essaient de produire des hybrides entre la chèvre et le mouton afin de créer des troupeaux d'animaux possédant en commun les caractères zootechniques les plus intéressants des deux espèces parentes. Bien que des rapports décrivant des hybrides vivants paraissent périodiquement dans les revues, les études effectuées au cours de la première moitié du $\mathrm{XX}^{\mathrm{e}}$ siècle ont montré que les fœus issus de tels accouplements survivent rarement au-delà de la huitième semaine de gestation (Berry, 1938; Warwick et al., 1935). Dans la plupart des cas, de tels « hybrides 
vivants " sont des chevreaux ressemblant à des agneaux ou des agneaux ressemblant à des chevreaux et l'examen cytogénétique, en mettant en évidence les compléments haplö̈des de chacun des deux parents, permet de déterminer leurs origines. Cependant quelques cas d'animaux de caryotype connu issus d'accouplement chèvre $\times$ mouton ont été observés et décrits dans la littérature (Bratanov et al., 1972 ; Bratanov et al., 1980 ; BUNCH et al., 1976 ; ElDRIDGE et al., 1983).

Un nouveau cas d'hybride vivant chèvre $\times$ mouton a été récemment relaté (DENIS et al., 1987). Dans cette note, nous décrivons le caryotype du même animal et de son produit.

\section{Matériels et méthodes}

\section{A. Les animaux}

\section{L'hybride chèvre $\times$ mouton}

L'hybride, de sexe femelle, né en 1984 est le produit d'un accouplement fortuit entre une chèvre et un bélier. La mère saillie par un bouc, étant par ailleurs entretenue en stabulation avec deux béliers (un Bleu du Maine et un Berrichon du Cher), a été également saillie par l'un d'eux. Elle a mis bas trois produits; deux d'entre eux sont morts dès les premiers jours après la naissance et ressemblaient à des chevreaux, tandis que le troisième présentait un aspect inhabituel.

L'hybride présentait certains traits incontestablement caprins avec un port de la tête et des oreilles, un comportement et une durée du cycle œstral de 21 jours. A trois ans, il toisait $0,71 \mathrm{~m}$ au garrot, avait une largeur de $0,30 \mathrm{~m}$, un tour de poitrine de $1,10 \mathrm{~m}$ et un poids de $85 \mathrm{~kg}$ (d'après QuitTet, 1980, des chèvres Alpines de même âge mesurent 0,70 à $0,80 \mathrm{~m}$, ont une largeur de poitrine de 0,16 à $0,18 \mathrm{~m}$, un tour de poitrine de 0,80 à $1,10 \mathrm{~m}$ et pèsent entre 50 et $80 \mathrm{~kg}$ ).

\section{Le produit hybride $\times$ mouton}

Au cours de l'année, l'hybride a été sailli par un bélier Solognot et a mis bas deux mâles, l'un vivant, l'autre mort-né. A l'âge de 18 mois, le produit ressemblait à un mouton de race rustique, assez enlevé et piètrement conformé. La toison était plus envahissante que chez la mère et n'a pas mué. On pouvait noter la présence d'un cornage franchement ovin. Les deux testicules étaient hypoplasiques mais de forme normale.

\section{B. Méthodes cytogénétiques}

Les caryotypes de l'hybride et de son produit ont été établis à partir de cultures de sang périphérique, effectués selon la technique de DE Grouchy et al. (1964). Du sang entier hépariné a été distribué à raison de $0,5 \mathrm{ml}$ par tube contenant $10 \mathrm{ml}$ de mélange nutritif HAM'S F 12 supplémenté de $20 \%$ de sérum fœetal bovin, de $25 \mu \mathrm{g} / \mathrm{ml}$ de 
concanavaline $\mathrm{A}$ et de $100 \mathrm{UI} / \mathrm{ml}$ d'antibiotique. Les cultures ont ensuite été mises à incuber à $37^{\circ} \mathrm{C}$ pendant trois jours. Après un blocage des mitoses de $1 \mathrm{~h} 30 \mathrm{~min}$ avec de la colcémide (concentration finale $0,09 \mu \mathrm{g} / \mathrm{ml}$ ), un choc hypotonique de $10 \mathrm{~min}$ à $37^{\circ} \mathrm{C}$ dans du chlorure de potassium $(0,56 \%)$ et trois fixations d'une heure dans un mélange d'acide acétique-éthanol (1:3), les suspensions cellulaires ont été étalées sur lame, séchées à la flamme, puis traitées pour les bandes G (GTG) suivant une technique proche de celle de Seabright (1971). Pour le marquage R (RBA) du 5bromodéoxyuridine (BrdU, $20 \mu \mathrm{g} / \mathrm{ml}$ ) a été ajouté dans certains tubes de culture huit heures avant le début du traitement. Les lames, préparées à partir de ces cultures, ont ensuite été colorées dans une solution d'acridine orange (Dutrillaux et al., 1973) et observées en lumière UV à l'aide d'un microscope Leitz.

\section{Résultats}

La photographie de l'hybride est représentée en figure 1. Le caryotype de l'hybride comprend 57 chromosomes dont 3 métacentriques et 54 acrocentriques. Les marquages $\mathrm{G}$ et $\mathrm{R}$ ont permis d'apparier et d'identifier chaque chromosome (fig. 2 et 3 ) ; les chromosomes de "mouton " sont à gauche et ceux de "chèvre " à droite. Seuls les chromosomes 15-15 et 23-24 présentent de légères différences eǹ marquage $R$.

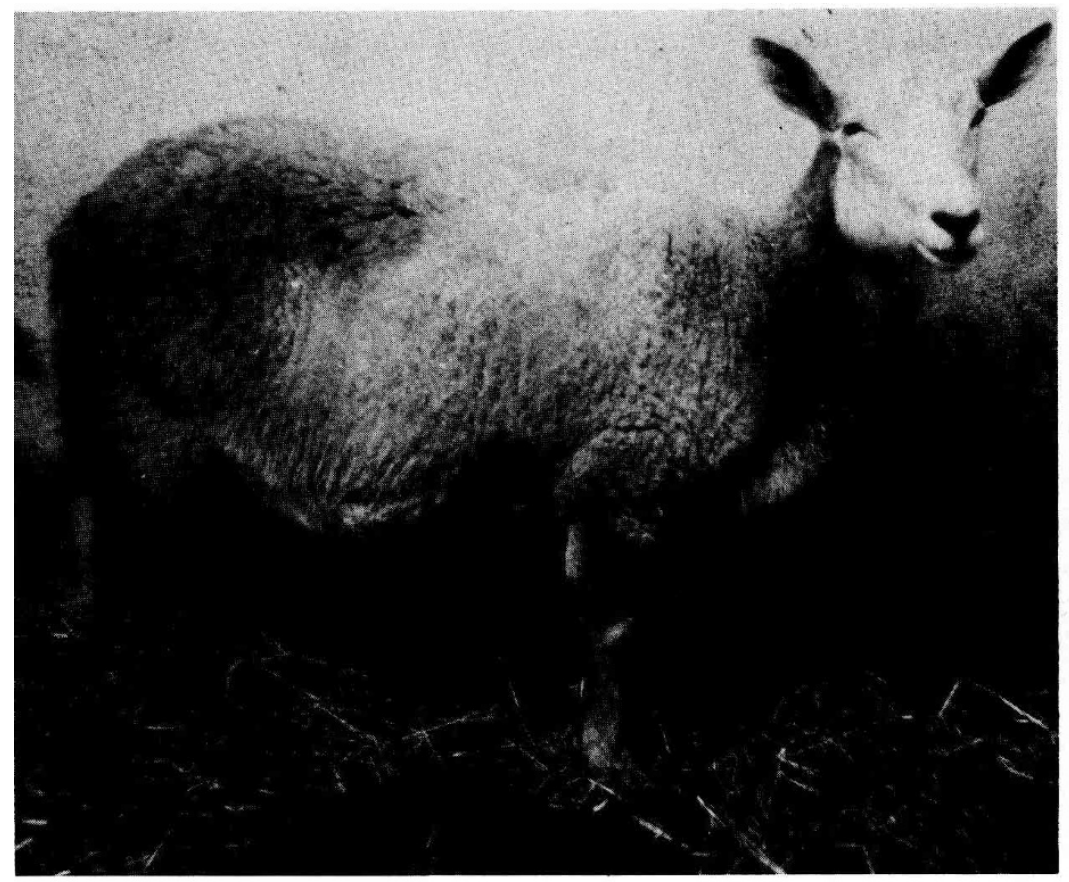

FIG. 1

Photographie de l'hybride chèvre $\times$ mouton.

Photograph of the goat $\times$ sheep hybrid. 

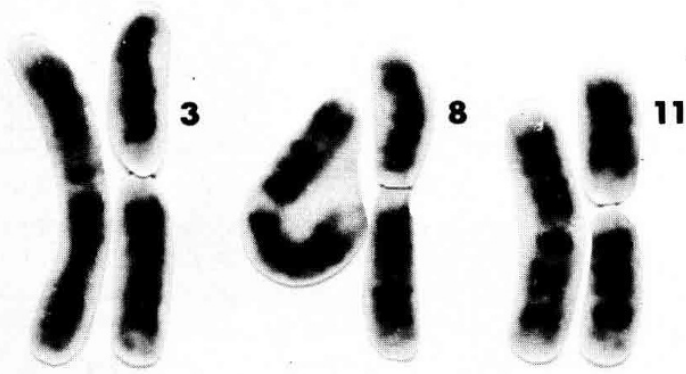

11

22

35
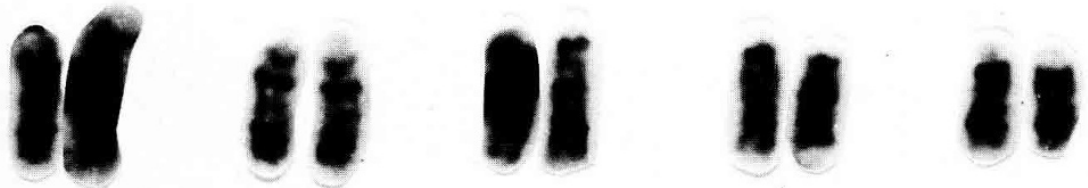

44

57

66

$7 \quad 10$

814
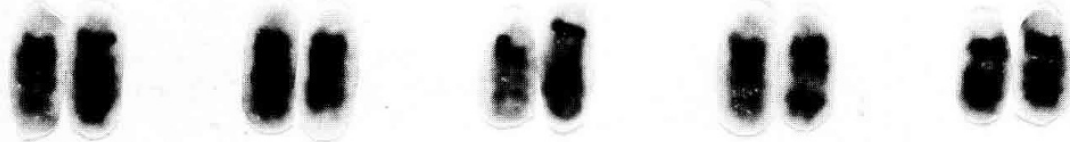

99

1012

$11 \quad 19$

1216

1313
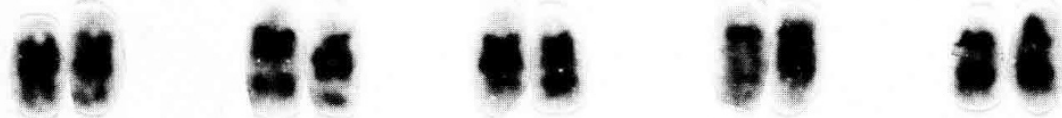

$14 \quad 18$

1515

1620

1717

1821
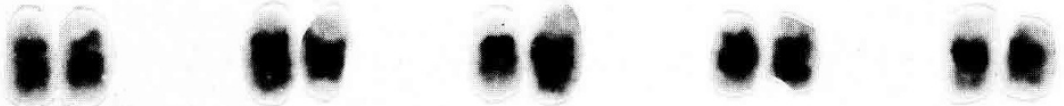

1922

2023

2125

2226

2324
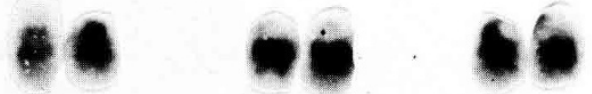

2528

2627

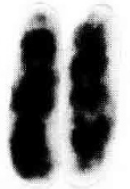

X $x$

FIG. 2

Caryotype de l'hybride en "bandes $G$ " (GTG).

Les chromosomes du mouton sont à gauche et ceux de la chèvre à droite.

$G$-banded karyotype of the goat $\times$ sheep hybrid.

The sheep chromosomes are on the left and the goat chromosomes on the right. 


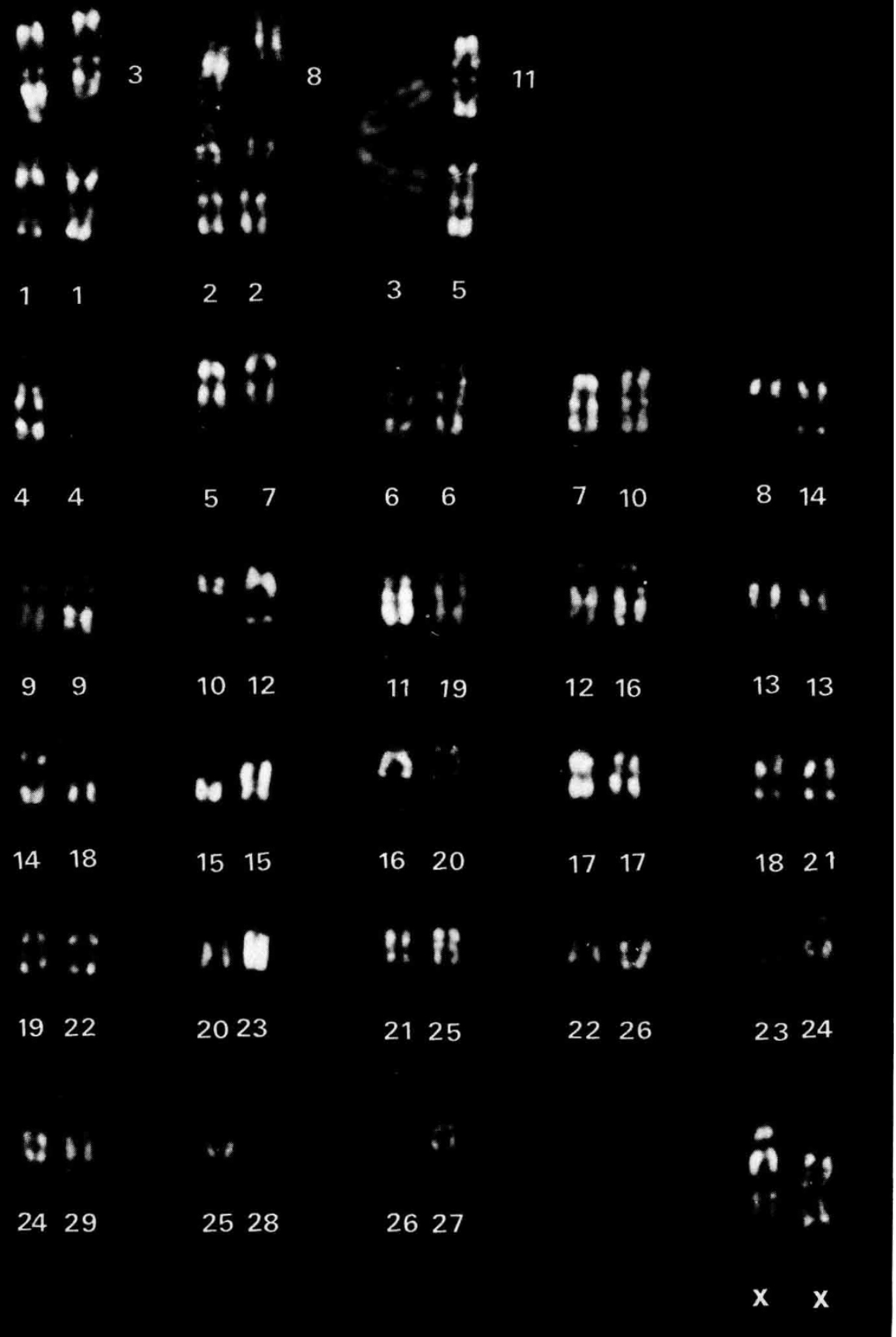

FIG. 3

Caryotype de l'hybride en "bandes R" (RBA).

Les chromosomes du mouton sont à gauche et ceux de la chèvre à droite.

$R$-banded karyotype of the goat $\times$ sheep hybrid.

The sheep chromosomes are on the left and the goat chromosomes on the right. 
Le caryotype du produit est composé de 54 chromosomes dont 6 métacentriques, 46 acrocentriques, un $X$ acrocentrique et un $Y$ métacentrique (fig. 4).

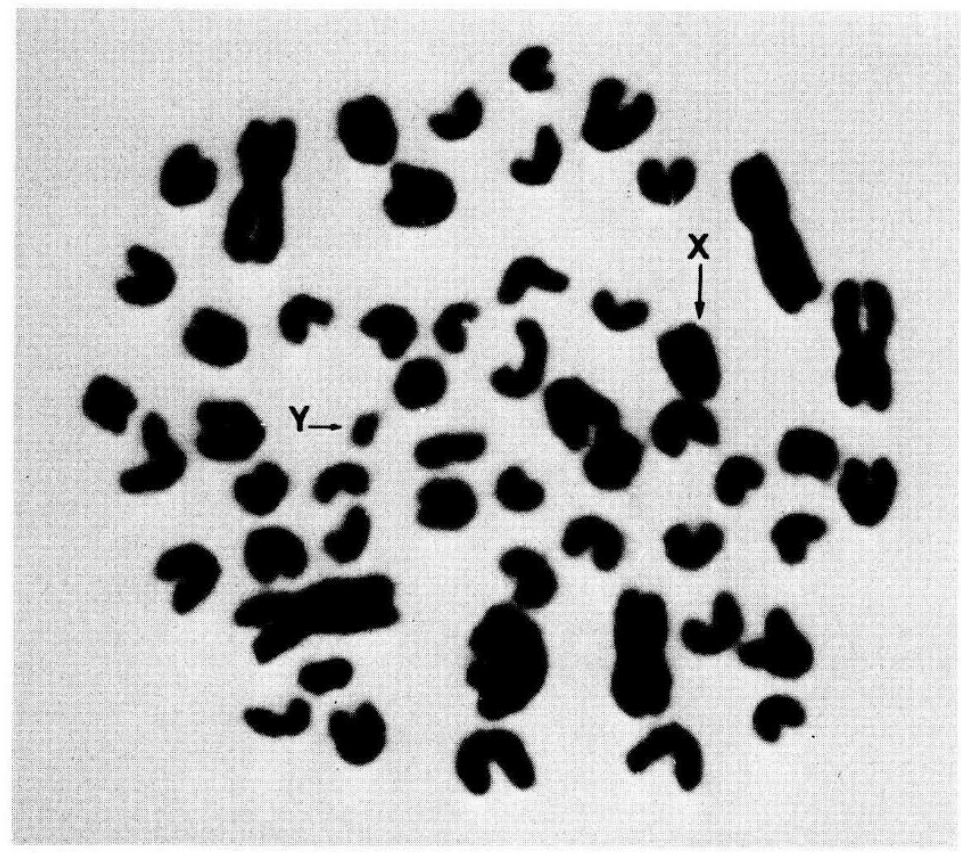

Fig. 4

Métaphase en coloration conventionnelle du produit du croisement de l'hybride par un bélier. Les chromosomes sexuels sont marqués par des flèches.

Giemsa-stained metaphase of male offspring from female sheep $\times$ goat hybrid mated to a ram. The sexual chromosomes are indicated by arrows.

\section{Discussion}

La principale difficulté des caryotypes de chèvre et de mouton est due surtout à l'absence de différences morphologiques entre la plupart des chromosomes qui les composent. En effet, la chèvre (Capra hircus) possède 58 autosomes acrocentriques, un gonosome $\mathrm{X}$ acrocentrique et un minuscule $\mathrm{Y}$ métacentrique et le mouton, 54 chromosomes parmi lesquels 6 sont métacentriques, 46 acrocentriques, un $X$ acrocentrique et un $\mathrm{Y}$ petit et métacentrique (pour revue CribiU \& Lherm, 1986 et CribiU \& MatejKa, 1985). La majorité des paires chromosomiques de ces deux espèces forme donc une série de taille décroissante dont les différences sont indiscernables entre deux paires consécutives. Seules, les techniques de marquage actuelles permettent, en induisant des bandes spécifiques sur chaque chromosome, de les reconnaître et de les apparier. Les caryotypes standard en bandes $G$ de ces deux espèces ont été tout d'abord proposés et 
adoptés à la Conférence de Reading, 1976 (Ford et al., 1980), puis modifiés et améliorés par LoNg (1985) et MATEJKA \& CribIU (1987) pour le mouton et par CRIBIU \& MATEJKA (1987a) pour la chèvre. Récemment, les caryotypes standard en bandes $\mathrm{R}$ (RBA) ont été effectués par MatejKa et al., (1988) pour le mouton et par CRIBIU \& MatejKA (1987b) pour la chèvre.

Le squelette assez grossier et les masses musculaires développées de l'hybride évoquent un type intermédiaire entre le mouton et la chèvre qui est confirmé par le caryotype. Celui-ci, en effet, est composé des compléments haploïdes de la chèvre $(n=30)$ et du mouton $(n=27)$ (fig. 4). Le dessin des bandes des bras chromosomiques révèlent des homologies, suggérant que ces deux espèces ne diffèrent que par trois translocations robertsoniennes et deux paires chromosomiques (15-15 et 23-24) qui ne semblent pas suffisantes pour entraîner la stérilité de notre hybride et de ceux vivants déjà décrits dans la littérature (Bratanov et al., 1972 ; Bunch et al., 1976).

Les hybrides vivants sont très rares et leur survie dépend du sens de croisement ; les brebis sont rarement fertilisées par les boucs et les quelques zygotes obtenus ne dépassent pas les premières divisions de segmentation; par contre, les chèvres sont plus fréquemment fécondées par les béliers, mais les fœetus sont avortés avant la huitième semaine. Les causes possibles de cette mortalité ne sont pas connues et ont été imputées aux différences immunologiques, hormonales et caryologiques des deux espèces parentes (pour revue McGoverN, 1969).

\section{Conclusion}

On pense généralement que les espèces de genre Capra et Ovis ont pour ancêtre commun un rubicapriné de 60 chromosomes. Si le genre Capra (Capra ibex, Capra falconeri et Capra hircus) a gardé le même nombre de chromosomes $(2 n=60)$, par contre le genre Ovis (Ovis canadensis, Ovis orientalis et Ovis aries) a subi une série de translocations robertsoniennes entraînant ainsi une réduction du nombre de chromosomes $(2 n=54)$ sans changement du nombre de bras chromosomiques (nombre fondamental). Ainsi il apparaît probable que l'acquisition des mécanismes d'isolement reproducteur soit due à l'accumulation de mutations géniques au cours du temps et que des types plus primitifs du mouton soient capables d'hybridation réussie avec la chèvre parce qu'ils partagent encore certains allèles communs contrôlant la croissance et le développement du fœtus. 


\section{Références bibliographiques}

Berry R.O., 1938. Comparative studies on the chromosomes number in sheep, goat and sheepgoat hybrids. J. Hered., 29, 343-350.

Bratanov K.T., Somlev B.P., Tsoncheva V.E., 1972. A study on the chromosomes of sheep $\times$ goat hybrids. C.R. Acad. Bulg. Sci., 25, 1109-1112.

Bratanov K., Dikov V., Somlev P., Efremova V., 1980. Chromosome complement and fertility of sheep $\times$ goat hybrids. In: $4^{\text {th }}$ European colloquium on cytogenetics of domestics animals, Uppsala, June 10-13, 1980, 262-266, Swedish University of Agricultural Sciences, Uppsala.

Bunch T.D., Foote W.P., SpILlet J.J., 1976. Sheep-Goat hybrid karyotypes. Theriogenology, 6, 379-385.

CRIBIU E.P., Lherm C., 1986. Caryotype normal et anomalies chromosomiques de la chèvre domestique (Capra hircus). Rec. Med. Vet., 162, 163-167.

CribiU E.P., MatejKa M., 1985. Caryotype normal et anomalies chromosomiques du Mouton domestique. Rec. Med. Vet., 161, 61-68.

Cribiu E.P., MatejKa M., 1987a. Idiogram and standardized G-band karyotype of the goat (Capra hircus). Zuchthyg., 22, 1-7.

Cribiu E.P., Matejka A., 1987b. Standardized R-band karyotype of the goat (Capra hircus). Zuchthyg, 22, 260-266.

De Grouchy J., Roubin P., Passage E., 1964. Microtechnique pour l'étude des chromosomes humains à partir d'une culture de leucocytes sanguins. Ann. Génét., 7, 45.

Denis B., Malher X., Seegers H., Dare R., Berland H.M., 1987. Note sur et à propos d'un hybride chèvre $\times$ mouton fertile. Elev. Insémin., 218, 3-10.

Dutrillaux B., Laurent C., Couturier J., Lejeune J., 1973. Coloration des chromosomes humains par l'acridine orange, après traitement par la 5-brombdéoxyuridine. C.R. Acad. Sci., Sér. D., 276, 3179-3182.

Eldridge F., Leipold W., Harris N., 1983. Sheep $\times$ goat hybrid : an additional case. J. Dairy Sci., 66, 253.

Ford C.E., Pollock D.L., Gustavsson I., 1980. Proceedings of the first international conference for the standardization of banded karyotypes of domestic animals, Reading, August 2-6, 1976. Hereditas, 92, 145-162.

LONG S.E., 1985. Standard nomenclature for the G-band karyotype of the domestic sheep (Ovis aries): Committee for standardized karyotype of Ovis aries. Hereditas, 103, 165-170.

Matejka M., Cribiu E.P., 1987. Idiogramme et représentation schématique des bandes $G$ des chromosomes du mouton domestique (Ovis aries L.). Génét. Sél. Evol., 19, 113-126.

Matejka M., Cribiu E.P., Popescu C.P., 1988. Diagrammatic représentation of RBA-banded karyotype of domestic sheep (Ovis aries L.). J. Hered. (sous presse).

Mc Govern P.T., 1969. Goat and sheep hybrids. Anim. Breed. Abstr., 37, 1-11.

QuitTet E., La chèvre, guide de l'éleveur. 288 p., La Maison Rustique, Paris.

SEABright M., 1971. A rapid banding technique for human chromosomes. Lancet, 2, 971-972.

Warwick B.L., Berry R.O., Horlacher W.R., 1935. Results of mating rams to Angora female goats. Proc. Am. Soc. Anim. Prod., 27, 225. 